\title{
Use of Diagnostic Laparoscopy in a Patient with Gastric Pneumatosis and Portal Venous Gas
}

\author{
Nilay R. Shah ${ }^{a} \quad$ Deborah S. Dossick ${ }^{a} \quad$ James A. Madura ${ }^{a}$ \\ Jacques P. Heppell ${ }^{b}$ \\ Divisions of ${ }^{a}$ General and Gastrointestinal Surgery and ${ }^{b}$ Colorectal Surgery, Department of \\ Surgery, Mayo Clinic Arizona, Phoenix, Ariz., USA
}

\section{Key Words}

Gastric pneumatosis · Diagnostic laparoscopy · Colorectal cancer · Computed tomography

\begin{abstract}
Gastric pneumatosis is a radiographic finding that represents a spectrum of conditions ranging from benign disease to abdominal sepsis and death. Along with portal venous gas, it is generally considered an ominous sign prompting emergent operative intervention. We report a rare case showing that diagnostic laparoscopy can be used to confirm or refute full thickness ischemic necrosis and that conservative management can be considered in some patients, recognizing the possibility of a benign process.
\end{abstract}

\section{Introduction}

Gastric pneumatosis results from infiltration of gas into the wall of the stomach. It is similar to pneumatosis intestinalis in the intestine. However, the stomach is very resistant to ischemia because of rich vascular supply. Intrusion of the gas into the bowel wall is due to mucosal or immune compromise. Acute massive gastric dilatation with intragastric pressure $>30 \mathrm{~cm} \mathrm{H}_{2} \mathrm{O}$ has been shown to decrease intramural blood flow, leading to necrosis [1]. Traditionally, pneumatosis of the intestine has been considered an ominous sign suggesting bowel ischemia with risk of infarction. However, many case reports have been published on pneumatosis suggesting that pneumatosis is a radiographic finding representing a spectrum of conditions [2] ranging from benign disease to abdominal sepsis and death. Various causes of pneumatosis in patients of various ages are inflammatory bowel disease, bowel distention, diverticulitis, chronic obstructive pulmonary disease, connective tissue disease, AIDS, ste- 
Shah et al:: Use of Diagnostic Laparoscopy in a Patient with Gastric Pneumatosis and Portal Venous Gas

roids, chemotherapy, pyloric stenosis, gastric outlet obstruction, duodenal stenosis, duodenal ulcers or tumors, etc. [3, 4]. Gastric pneumatosis can be a life-threatening gangrenous gastric pneumatosis (emphysematous gastritis) or benign non-gangrenous gastric pneumatosis (gastric emphysema).

The finding of gastric pneumatosis can signify a multitude of possibilities both in terms of causes and consequences. Generally, gangrenous gastric pneumatosis requires emergency surgery, whereas non-gangrenous gastric pneumatosis may be managed by non-operative decompression. The mortality rate averages $41 \%$ in adults and $6 \%$ in children [5]. However, many recent case reports have concluded that the significance of pneumatosis depends upon the underlying clinical condition and physical findings. There are no case reports in the literature suggesting utilization of laparoscopy to determine full thickness necrosis in patients with radiological diagnosis of pneumatosis and esophagogastroduodenoscopy showing mucosal necrosis. However, we report a case of gastric pneumatosis which emphasizes the importance of diagnostic laparoscopy to rule out full thickness necrosis in patients with gastric pneumatosis, which can ultimately avoid major surgery such as gastrectomy.

\section{Case Report}

A vigorous 84-year-old man presented with recurrent adenocarcinoma and a solitary hepatic metastasis several years after laparoscopic right hemicolectomy. A wedge excision of a $1.8 \mathrm{~cm}$ lesion in segment 7 of the liver was performed after resecting en bloc a $3.5 \mathrm{~cm}$ mass at the ileo-colonic anastomosis that invaded the retroperitoneum and the serosa of the third portion of the duodenum.

After recovering from adynamic ileus, the patient was sent home on postoperative day 9 to return soon after with nausea and severe vomiting. On arrival, he had normal vital signs except for tachycardia that resolved after rehydration. Placement of a nasogastric tube returned $150 \mathrm{ml}$ of clotted blood. His abdomen was mildly distended and non-tender to palpation. He had normal labs except for leukocytosis (WBC: 20,400). An abdominal X-ray was performed and showed an atypical gas pattern involving the stomach. There were also signs of gastric pneumatosis and portal venous gas. Computed tomography of the abdomen with oral and intravenous contrast was obtained (fig. 1) which showed gastric distention and pneumatosis with portal venous gas. Esophagogastroduodenoscopy confirmed the gastric mucosal findings (fig. 2). It showed areas of mucosal color changes in patchy distribution, along with edematous and friable areas suggesting necrosis. Biopsy was not obtained because of concern of full thickness necrosis and perforation.

Gastric pneumatosis with portal venous air and endoscopic concern for gastric ischemia prompted urgent diagnostic laparoscopy. This demonstrated completely normal-appearing gastric serosa with no signs suggesting full thickness infarction or distal obstruction (fig. 3). The patient was treated conservatively with intravenous antibiotics covering aerobic and anaerobic bacteria, nasogastric decompression and parenteral nutrition. He recovered well with this conservative approach and was discharged home in a stable condition. On followup, he was able to tolerate a regular diet.

\section{Discussion}

In the 1940s and 1950s, there were few reports of emphysematous gastritis with a mortality rate of $60-80 \%[6,7]$. Various terminologies have been used to describe air in the 
Shah et al.: Use of Diagnostic Laparoscopy in a Patient with Gastric Pneumatosis and Portal Venous Gas

wall of the stomach, including cystic pneumatosis, interstitial gastric emphysema and emphysematous gastritis [8]. Gastric pneumatosis is a generalized term that includes any cause of air in the wall of the stomach. Since then, multiple case reports of gastric pneumatosis have been published with varied outcomes, ranging from conservative management to operative interventions ultimately leading to total gastrectomy [9-14].

Gastric pneumatosis is a radiographic finding that represents a spectrum of conditions [2] ranging from benign disease to abdominal sepsis and death. Four theories have been proposed: (1) bacterial - intramucosal gas is generated by gas-forming bacilli; (2) mechanical - increased intraluminal pressure during endoscopy or obstruction; (3) mucosal damage - air enters through disrupted mucosa due to gastric ulcer; (4) pulmonary disease alveolar air dissects down through the mediastinum within the gastric wall $[2,15]$. In our patient, the most likely reason for pneumatosis was mechanical due to retching and severe vomiting in the absence of duodenal obstruction which was confirmed by upper gastrointestinal Gastrografin.

Gastric pneumatosis with portal venous gas is generally considered an ominous sign prompting emergent operative intervention. However, the outcome depends on multiple factors. Management should be guided by the proposed mechanism and clinical signs as opposed to radiographic and endoscopic findings alone. Therefore, diagnostic laparoscopy to confirm or refute full thickness ischemic necrosis and conservative management can be considered in some patients, recognizing the possibility of a benign process [16].

\section{Conclusion}

Diagnostic laparoscopy should be considered as an essential tool to confirm or refute full thickness ischemic necrosis in patients with gastric pneumatosis so that extremely morbid procedures such as total gastrectomy can be avoided.

\section{Disclosure Statement}

None of the authors have any conflict of interest to declare.

\section{References}

1 Lunca S, Rikkers A, Stanescu A: Acute massive gastric dilatation: severe ischemia and gastric necrosis without perforation. Rom J Gastroenterol 2005;14:279-283.

-2 St Peter SD, Abbas MA, Kelly KA: The spectrum of pneumatosis intestinalis. Arch Surg 2003;138:68-75.

3 Kataria R, Bhatnagar V, Wadhwa S, Mitra DK: Gastric pneumatosis associated with preduodenal portal vein, duodenal atresia, and asplenia. Pediatr Surg Int 1998;14:100-101.

-4 Kawano S, Tanaka H, Daimon Y, Niizuma T, Terada K, Kataoka N, Iwamura Y, Aoyama K: Gastric pneumatosis associated with duodenal stenosis and malrotation. Pediatr Radiol 2001;31:656-658.

5 Lim RK, McKillop S, Karanicolas PJ, Scott L: Massive gastric pneumatosis from pyloric stenosis. CMAJ 2010;182:E227.

6 Weens HS: Emphysematous gastritis. Am J Roentgenol Radium Ther 1946;55:588-593.

7 Henry GW: Emphysematous gastritis. Am J Roentgenol Radium Ther Nucl Med 1952;68:15-18.

8 Colquhoun J: Intramural gas in hollow viscera. Clin Radiol 1965;16:71-86.

-9 Bhargava P, Parisi M: Gastric pneumatosis and portal venous gas: benign findings in hypertrophic pyloric stenosis. Pediatr Radiol 2009;39:413.

10 Abboud B, Mchayleh W, Sleilaty G, Yaghi C: Gastric pneumatosis as a manifestation of ischemic infarction of the stomach. Report of a case and review of the literature. J Med Liban 2006;54:217-220. 
Shah et al.: Use of Diagnostic Laparoscopy in a Patient with Gastric Pneumatosis and Portal Venous Gas

11 Pauli EM, Tomasko JM, Jain V, Dye CE, Haluck RS: Multiply recurrent episodes of gastric emphysema. Case Rep Surg 2011;2011:587198.

12 Lewin M, Pocard M, Caplin S, Blain A, Tubiana JM, Parc R: Benign hepatic portal venous gas following caustic ingestion. Eur Radiol 2002;12(suppl 3):S59-S61.

13 Kingsley DD, Albrecht RM, Vogt DM: Gastric pneumatosis and hepatoportal venous gas in blunt trauma: clinical significance in a case report. J Trauma 2000;49:951-953.

14 Kim SJ, Cho H, Lee SW, Choi SH, Hong YS: Gastric pneumatosis and acute massive gastric dilatation without specific cause. J Emerg Med 2013;44:111-113.

15 Johnson PT, Horton KM, Edil BH, Fishman EK, Scott WW: Gastric pneumatosis: the role of CT in diagnosis and patient management. Emerg Radiol 2011;18:65-73.

-16 Wiesner W, Mortelé KJ, Glickman JN, Ji H, Ros PR: Pneumatosis intestinalis and portomesenteric venous gas in intestinal ischemia: correlation of CT findings with severity of ischemia and clinical outcome. AJR Am J Roentgenol 2001;177:1319-1323.

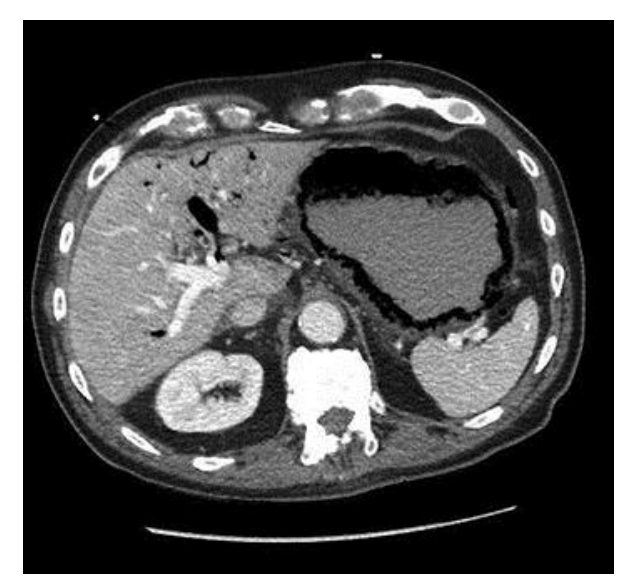

Fig. 1. Computed tomography of the abdomen with oral and intravenous contrast showed gastric distention and pneumatosis with portal venous gas.

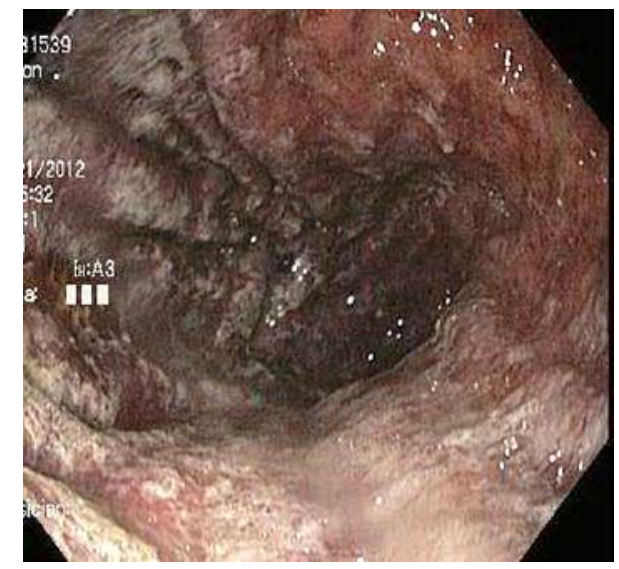

Fig. 2. Esophagogastroduodenoscopy confirmed the gastric mucosal findings with necrosis. 
Case Reports in
Gastroenterology
Case Rep Gastroenterol 2013;7:261-265

DOI: $10.1159 / 000351876$

Shah et al:: Use of Diagnostic Laparoscopy in a Patient with Gastric Pneumatosis and Portal Venous Gas

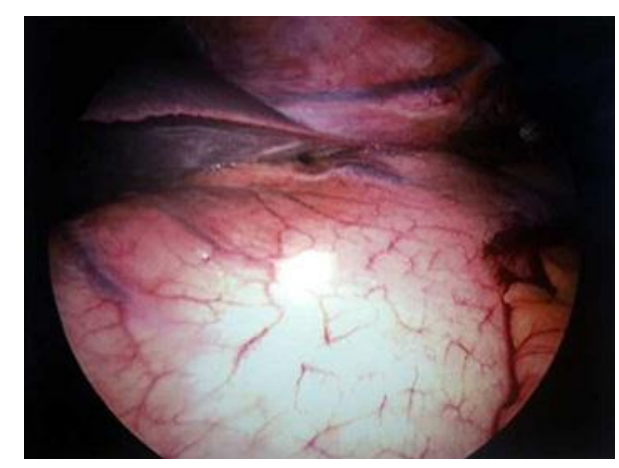

Fig. 3. Urgent diagnostic laparoscopy demonstrated completely normal-appearing gastric serosa with no signs suggesting full thickness infarction or distal obstruction. 